\title{
Transperitoneal and retroperitoneal approach in laparoscopic partial nephrectomy for posterior cT1 renal tumors: A retrospective, two-centers, comparative study
}

\author{
Nikolaos Ferakis ${ }^{1}$, Antonios Katsimantas ${ }^{1}$, Nikolaos Charalampogiannis ${ }^{2}$, Spyridon Paparidis ${ }^{1}$, \\ Jens Jochen Rassweiler ${ }^{2}$, Ali Serdar Gözen ${ }^{2}$ \\ ${ }^{1}$ Department of Urology, Korgialenio-Benakio Hellenic Red Cross Hospital, Athens, Greece; \\ ${ }^{2}$ Department of Urology, SLK-Kliniken Heilbronn, University of Heidelberg, Heilbronn, Germany.
}

\begin{abstract}
Summary Objectives: To compare perioperative, onco-
logical and functional outcomes of Laparoscopic Transperitoneal Partial Nephrectomy (LTPN) and Retroperitoneal Laparoscopic Partial Nephrectomy (LRPN) for posterior, cT1 renal masses (RMs).

Materials and methods: Databases of two urologic institutions applying different laparoscopic surgical approaches on posterior cT1 RMs between June 2016 and November 2018 were retrospectively evaluated. Data on patient demographics, perioperative data and tumor histology were collected and further analyzed statistically.

Results: Each group consisted of 15 patients. Baseline characteristics were comparable in each group. When compared to LTPN, LRPN was associated with significantly shorter operative time $(O T)$ (115 min versus $199 \mathrm{~min}, p<0.05)$. No significant differences were detected in the other outcomes.

Conclusions: LRPN is associated with a significantly shorter OT compared to LTPN for posterior cT1 RMs. Both surgical approaches are safe, feasible and credible, demonstrating optimal results.
\end{abstract}

KEY WORDS: Laparoscopy; Partial nephrectomy; Transperitoneal; Retroperitoneal; Renal tumor.

Submitted 2 March 2020; Accepted 15 March 2020

\section{INTRODUCTION}

Partial nephrectomy (PN) is as a valid surgical approach for cTl renal masses (RMs) and is strongly recommended whenever technically feasible (1-6). It can equally be performed by open (OPN), pure laparoscopic (LPN) or robot-assisted laparoscopic approach (3, 7-9).

A wide variety of endoscopic instruments and the steadily increasing laparoscopic experience rendered LPN a feasible treatment modality for cTl RMs (1, 7, 10-11). Both routes, either transperitoneal (TP) or retroperitoneal (RP), can be equally advocated $(1,7,11-15)$.

A benefit of retroperitoneal LPN (LRPN) is the direct, rapid access to the posterior hilar structures and to posterior RMs allowing for less kidney's mobilization and rotation $(1,7,12-18)$. LRPN avoids bowel mobilization, need for lysis of adhesions in patients with prior abdominal surgery and peritoneal cavity irritation through contamination of blood and urine $(1,3,7,11,14,15)$. Moreover, the RP space may tamponade a possible postoperative bleeding and avoids peritonitis caused by a possible postoperative urinary fistula $(5,16)$. The presence of abundant RP fat (e.g. in obese patient) or the case of a large or anteriorly located tumor may render LRPN challenging $(6,10,15,16)$. In addition, LRPN is technically demanding, with a steep learning curve (19). TP route offers better spatial orientation due to the presence of more familiar anatomic landmarks $(1,7,11,14$, $15,19)$. Ease in port placement due to larger skin surface, increased working space allowing for wider angulations and enhanced maneuverability are in favor of transperitoneal LPN (LTPN) (1, 3, 7, 11, 14, 15, 19). Major drawbacks of the LTPN are the difficulty in dissection of posterior RMs and subsequent reconstructive suturing, which may guide surgeon's decision to perform OPN instead of minimal invasive procedure, if he is not familiar with the steps for creating RP space $(15,17,18)$. The aim of the present study is to analyze and present comprehensively the perioperative, oncological and functional outcomes of LTPN and LRPN for posterior cT1 RMs in two urologic centers.

\section{Materials ANd Methods}

Following institutional review board approval, we reviewed prospectively collected data of two centers to evaluate consecutive patients who underwent LTPN or LRPN for posterior, cTl RM from June 2016 to November 2018. All TP procedures (Group A) were performed by one experienced surgeon (NF) at a single institution in Greece, where LTPN is the standard operative technique for cTl RMs. All RP procedures (Group B) were performed by two experienced surgeons (JJR, ASG) at a large German academic center, where LRPN is the usual operative technique for posterior RMs. Intraoperative findings were recorded systematically on surgical and video files and evaluated retrospectively. Inclusion and exclusion criteria are listed in Table 1. We excluded patients who underwent ETHOS chairassisted LRPN, as it was demonstrated that it significantly improves intraoperative parameters (16).

Preoperatively, all patients underwent routine laboratory testing, chest x-Ray, cardiological evaluation and abdominal Computed Tomography scan or Magnetic Resonance Imaging. Informed consent was obtained by each patient. 
Table 1.

Inclusion and exclusion criteria.

\begin{tabular}{|c|}
\hline $\begin{array}{l}\text { Inclusion } \\
\text { Single, unilateral, contrast-enhanced cT1NOMO renal mass } \\
\text { Localization of the tumor in the posterior renal surface } \\
\text { Resection of the mass via transperitoneal or retroperitoneal laparoscopic partial } \\
\text { nephrectomy }\end{array}$ \\
\hline $\begin{array}{l}\text { Exclusion } \\
\text { Conversion to open partial or radical nephrectomy } \\
\text { LRPN using the ETHOS chair } \\
\text { Missing data }\end{array}$ \\
\hline
\end{tabular}

Our database included information on patient's age, gender, body mass index (BMI), American Society of Anesthesiologists (ASA) score, tumor characteristics, preand post-operative serum hemoglobin $(\mathrm{Hb})$ value, preand post-operative estimated glomerular filtration rate (eGFR) according to the Modification of Diet in Renal Disease, tumor complexity evaluated by Preoperative Aspects and Dimensions Used for an Anatomical Classification (PADUA) score, operative time (OT), warm ischemia time (WIT), length of hospital stay (LOS), TNM stage and histology, surgical margins (SM) status [positive SM (PSM) defined as the presence of cancer cells at the level of the inked parenchymal excision surface] and intra-/post-operative complications (according to the modified Clavien-Dindo classification scale).

The technique of LRPN has been described previously (16). In brief, under general anesthesia and in a full flank position, a RP space is created through a $16-18 \mathrm{~mm}$ transverse incision below the tip of the $12^{\text {th }}$ rib in the "musclefree' Petit's triangle. The RP space is entered bluntly by using the index finger and developed by using an optical dilatation balloon. A $12 \mathrm{~mm}$ trocar is placed with the guidance of index finger inside the RP space for the right hand and a $5 \mathrm{~mm}$ trocar for the left hand. Optionally, another $5 \mathrm{~mm}$ trocar is placed under view medially to the rim of peritoneum when a retraction of the kidney and Gerota's fascia (GF) is needed. GF is incised horizontally parallel to the psoas muscle and the renal hilum is accessed for the dissection of the renal artery. The entire kidney is isolated from perirenal fat (except from the fat overlying the tumor) to localize the RM. The kidney is mobilized and the incision line around the tumor is scored with bipolar forceps. We clamp the renal artery with a bulldog clamp and we proceed to tumor's excision according to the principles of enucleo-resection, preserving a $5-\mathrm{mm}$ rim of normal renal tissue. The tumor is placed in an endoscopic specimen bag. Subsequently, we perform inner renorrhaphy by continuous suturing with a barbed suture (V-LocTM, Covidien, SH, $20 \mathrm{~cm}$ ) preloaded with an absorbable LAPRA-TY ${ }^{\circledR}$ clip supported by a Vicryl patch or Durapatch, which is stitched out on the contralateral side of the resection wound and fixed with a LAPRA-TY ${ }^{\circledR}$ clip. Renal parenchyma is closed with a continuous suture secured at every loop using LAPRA-TY ${ }^{\circledR}$ clips, the bulldog clamp is removed and the suturing line is covered with perirenal fat. Finally, the specimen bag is removed through the optic trocar incision and is grossly inspected, a drain (24 Fr) is placed via $12 \mathrm{~mm}$ port incision and port incisions are closed.
Regarding LTPN, the patient is fixed in the lateral flank position with the table half flexed, under general anesthesia. We use open Hasson technique to place the first trocar $(12 \mathrm{~mm})$ for the $30^{\circ}$ laparoscope 2 fingerbreadths above and $8-10 \mathrm{~cm}$ lateral to the umbilicus. We place the rest of trocars (one $12 \mathrm{~mm}$ and one $5 \mathrm{~mm}$ at the midclavicular line according to the triangulation principle, serving as surgeon's working channels, and one $12 \mathrm{~mm} 3$ fingerbreadths medial to the superior anterior iliac spine and one $5 \mathrm{~mm} 8 \mathrm{~cm}$ lateral to the camera trocar for the first assistant) under direct vision. On the right side, we may place another $5 \mathrm{~mm}$ subxyphoid trocar to retract liver. We release colon's lateral attachments, to deflect it medially, and splenorenal or hepatorenal ligaments.

On the right side, we mobilize duodenum (Kocher maneuver) medially, until we clearly visualize vena cava. GF is opened, the genital vessels, the proximal ureter, the psoas muscle and the renal pedicle are located and the kidney is defatted down to the renal capsule (preserving the fat overlying the tumor) and is mobilized. After locating the tumor, the margin of resection is marked with electrocautery. We use Rummel Tourniquet (RT) technique on renal artery in order to achieve WI, similarly to the technique described by Shefler et al. (20). We create our tourniquet by using a $30 \mathrm{~cm}$ long $2 \mathrm{~mm}$ thick vessel loop, a $2 \mathrm{~cm}$ cylinder sheath prepared from a 16 Fr Levin tube and a large Hem-o-Lock clip.

The tumor is excised [(enucleo)-resection] using cold scissors at $5 \mathrm{~mm}$ from the tumor's edge. Subsequently, we perform inner renorrhaphy using a $15 \mathrm{~cm}$ 3-0 V-Loc running suture, we release RT (early unclamping technique) and we complete renorrhaphy using a $13 \mathrm{~cm} \mathrm{1/0} \mathrm{polyg-}$ lycolic acid running suture. We secure our running sutures using a large Hem-o-Loc clip at each exit point. We reapproximate perirenal fat over the cutting surface, the tumor is removed via a $12 \mathrm{~mm}$ port using an endoscopic specimen bag and is grossly inspected, a drain $(16 \mathrm{Fr})$ is placed via a port incision and port incisions are closed.

In both techniques, 2-Dimensional High Definition (HD) cameras were used, pneumo (retro) peritoneum was maintained at $12 \mathrm{mmHg}$ and mannitol iv was administered prior to clamping the renal artery. In both departments, we apply similar perioperative protocols: bowel preparation is applied preoperatively, no antiemetic or opioid drugs are routinely administered postoperatively, nasogastric tube is removed immediately postoperatively, patients receive liquid diet and are mobilized and Foley catheter is removed on the 1st postoperative day.

The patients are discharged following drain's removal and when they are medically stable, full ambulatory without assistance and need for intravenous analgesia and capable to tolerate a light diet. Patients with malignant pathology are scheduled for 6-month imaging follow-up.

All statistical analyses were performed using an SPSS25 statistical program. Data were less than $35(\mathrm{~A}=15, \mathrm{~B}=$ 15), so standard normality assumptions did not meet. Thus, non-parametric statistical tests were conducted. Quantitative characteristics were compared using MannWhitney test and qualitative characteristics were compared using 2-tailed chi-square test. A p value $<0.05$ was considered statistically significant. Median values and 
range were calculated for quantitative continuous variables and proportions for nominal variables.

\section{RESULTS}

Following exclusion, each cohort consisted of 15 patients. Preoperative characteristics of both groups (demographic data and tumors' characteristics) were comparable (Table 2).

Peri-operative data of both groups are summarized on Table 3. Variation of serum $\mathrm{Hb}$ value pre- and 10 hours post-operatively was comparable in both groups. However, 4 patients were transfused intra-operatively (1 unit of packed red blood cells each), being all of them from group A ( 3 pTla, lpTlb, 1 with no ischemia), although the difference between cohorts was not statistically significant. OT was shorter in group B $(p<0.05)$. Three patients of group A (1 pTla, 2 pTlb) and 5 patients of group B (3 pTla, 2 pTlb) underwent clampless LPN. Regarding postoperative complications, both groups were comparable. There were 5 patients of group A with minor postoperative complications (fever and bleeding which was managed conservatively with transfusion). One patient of group B presented prolonged drain excretion of serum fluid (biochemically confirmed). One patient of group A with a medical history of acute myocardial infarction died on the $4^{\text {th }}$ postoperative day, following an episode of orthostatic hypotension, which resulted in fall on the ground, head injury and heart attack. No patient needed antiemetic drugs postoperatively or readmission following discharge.

Oncological data are demonstrated on Table 4. PSM rate was $13.3 \%$ in each cohort. There was solitary, focal, microscopic invasion of the tumor pseudocapsule in 3 cases [Group A: 2 cases of cTlb, clear-cell Renal Cell Carcinoma (ccRCC), Fuhrman Grade (FG) 2 and 3, Group B: 1 case of cTlb, ccRCC, FG 2]. In addition, there was a case of pTla, papillary type 2 RCC in group B with equivocal SM status (focally), which was interpreted as positive. PSMs were detected only in cases of clampless $\mathrm{PN}$. There was no local or distant recurrence during the follow-up period.

Table 2.

Baseline characteristics of the two groups.

\begin{tabular}{|c|c|c|c|}
\hline & LTPN $\left(n_{A}=15\right)$ & LRPN $\left(n_{B}=15\right)$ & \\
\hline & \multicolumn{2}{|c|}{ Number } & $\mathrm{p}$ \\
\hline Gender ${ }^{a}$ - Male/female & $9 / 6$ & $13 / 2$ & 0.09 \\
\hline \multirow[t]{2}{*}{ Side - Right/left } & $5 / 10$ & $10 / 5$ & 0.07 \\
\hline & \multicolumn{2}{|c|}{ Median $\left(25^{\text {th }}-75^{\text {th }}\right.$ centile) } & $p$ \\
\hline $\mathrm{Age}^{\mathrm{b}}$ (years) & $70(62-73)$ & $68(53-75)$ & 0.95 \\
\hline $\mathrm{BMI}^{\mathrm{b}}\left(\mathrm{kg} / \mathrm{m}^{2}\right)$ & $25.0(24.2-27.3)$ & $28.1(26.1-30.8)$ & 0.05 \\
\hline ASA score ${ }^{b}$ & $2.0(1.0-2.0)$ & $2.0(2.0-3.0)$ & 0.140 \\
\hline Preop. $H b^{b}(g / d l)$ & $14.4(12.7-15.1)$ & $13.0(11.8-14.4)$ & 0.201 \\
\hline Preop. eGFR' $\left(\mathrm{ml} / \mathrm{min} / 1.73 \mathrm{~m}^{2}\right)$ & $76.3(69.7-85.1)$ & $68.0(63.8-87.8)$ & 0.458 \\
\hline PADUA score ${ }^{b}$ & $7.5(6.8-9.0)$ & $7.0(6.0-10.0)$ & 0.798 \\
\hline Tumor size $(\mathrm{cm})$ & $3.5(2.5-3.8)$ & $4.5(2.2-5.0)$ & 0.271 \\
\hline \multicolumn{4}{|c|}{$\begin{array}{l}\text { LTPN/LRPN: Laparoscopic Transperitoneal/Retroperitoneal Partial Nephrectomy; BMI: Body Mass Inde } \\
\text { ASA score: American Society of Anesthesiologists score; Preop.: Preoperative, Hb: Hemoglobin; } \\
\text { eGFR: estimated glomerular filtration rate; PADUA score: Preoperative Aspects and Dimensions Used } \\
\text { for an Anatomical Classification score. } \\
{ }^{2}{ }^{2} \text { test, Fisher's test: }{ }^{\circ} \text { Mann-Whitney test, } 2 \text {-sided Fisher exact test. }\end{array}$} \\
\hline
\end{tabular}

Table 3.

Perioperative data of the two groups.

\begin{tabular}{|c|c|c|c|c|}
\hline \multirow{3}{*}{$\overline{\text { Hb postop. }{ }^{\mathrm{a}}(\mathrm{g} / \mathrm{dl})}$} & \multicolumn{2}{|r|}{$\operatorname{LTPN}\left(n_{A}=15\right)$} & LRPN ( $\left.n_{B}=15\right)$ & \multirow[b]{2}{*}{$\mathrm{p}$} \\
\hline & & \multicolumn{2}{|c|}{ Median $\left(25^{\text {th }}-75^{\text {th }}\right.$ centile) } & \\
\hline & & $11.8(11.3-13.5)$ & $11.1(10.5-13.3)$ & 0.256 \\
\hline Variation of Hb pre- and postop. ${ }^{\mathrm{a}}(\mathrm{g} / \mathrm{dl})$ & & $1.4(0.8-3.4)$ & $1.35(0.675-1.925)$ & 0.646 \\
\hline \multicolumn{2}{|l|}{$\begin{array}{l}\text { Variation of eGFR preop. } \\
\text { and at discharge }\left(\mathrm{m} / \mathrm{min} / 1.73 \mathrm{~m}^{2}\right)\end{array}$} & $6.8(0.0-14.1)$ & $0.0(-5.0-13.8)$ & 0.352 \\
\hline \multicolumn{2}{|l|}{ Operative time ${ }^{a}$ (minutes) } & $99(150.0-220.0)$ & $115(100.0-180.0)$ & $<0.05$ \\
\hline \multirow{2}{*}{\multicolumn{2}{|c|}{$\begin{array}{l}\text { WIT (minutes) } \\
\text { Length of Hospital Stay (days) }\end{array}$}} & $16(14.0-20.0)^{*}$ & $23(20.0-28.5)^{* *}$ & 0.160 \\
\hline & & $5(4-7)$ & $6(5-6)$ & 0.233 \\
\hline & & \multicolumn{2}{|c|}{ Number } & $\mathrm{p}$ \\
\hline$\overline{W W^{b}}$ & Yes/No & $12 / 3$ & $10 / 5$ & 0.68 \\
\hline Intraoperative transfusion ${ }^{b}$ & Yes/No & $4 / 11$ & $0 / 15$ & 0.10 \\
\hline Postop. complications ${ }^{b}$ & Yes/No & $6 / 9$ & $1 / 14$ & 0.06 \\
\hline Minor complications (grade $1 \& 2)^{b}$ & Yes/No & $5 / 10$ & $1 / 14$ & 0.17 \\
\hline Postop. fever (\%) & & $2(13.3)$ & $(-)$ & \\
\hline Postop. bleeding-transfusion (\%) & & $3(20)$ & $(-)$ & \\
\hline Prolonged drain excretion (\%) & & $(-)$ & $1(6.7)$ & \\
\hline \multirow[t]{2}{*}{ Major complications (z grade 3$)^{b}$} & Yes/No & $1 / 14$ & $0 / 15$ & 0.31 \\
\hline & Death $(\%)$ & 1 & $(-)$ & \\
\hline
\end{tabular}

Table 4.

Oncological data of the two groups.

\begin{tabular}{|c|c|c|c|c|}
\hline \multirow{4}{*}{$\begin{array}{l}\text { Tumor's size }^{\mathrm{a}}(\mathrm{cm}) \\
\text { Follow-up period }{ }^{\text {(months) }}\end{array}$} & & LTPN $\left(n_{A}=15\right)$ & $\operatorname{LRPN}\left(n_{B}=15\right)$ & \multirow{3}{*}{$\begin{array}{c}\mathbf{p} \\
0.271\end{array}$} \\
\hline & & \multicolumn{2}{|c|}{ Median $\left(25^{\text {th }}-75^{\text {th }}\right.$ centile $)$} & \\
\hline & & $3.5(2.5-3.8)$ & $4.5(2.2-5.0)$ & \\
\hline & & $23.0(19.8-22.3)$ & $17.0(15.0-24.3)$ & 0.137 \\
\hline & & \multicolumn{2}{|c|}{ Number } & $\mathrm{p}$ \\
\hline Malignancy ${ }^{b}$ & Yes/No & $12 / 3$ & $14 / 1$ & 0.28 \\
\hline \multirow[t]{2}{*}{$S M^{b}$} & Neg./Pos. & $13 / 2$ & $13 / 2$ & 1.00 \\
\hline & & \multicolumn{2}{|c|}{ Number } & $\mathrm{p}$ \\
\hline Benign lesions & Oncocytoma & $3(20)$ & $1(6.7)$ & \\
\hline \multirow{7}{*}{ Malignant lesions } & $\operatorname{ccRCC}$ & $9(60)$ & $9(60)$ & \\
\hline & $\mathrm{FG} 1$ & $(-)$ & $5(55.6)$ & \\
\hline & $\mathrm{FG} 2$ & $6(66.7)$ & $4(44.4)$ & \\
\hline & $\mathrm{FG} 3$ & $2(22.2)$ & $(-)$ & \\
\hline & $\mathrm{FG} 4$ & $1(11.1)$ & $(-)$ & \\
\hline & pRCC type 2 & $1(6.7)$ & $4(26.7)$ & \\
\hline & $\operatorname{chRCC}$ & $2(13.3)$ & $1(6.7)$ & \\
\hline \multirow[t]{3}{*}{ T stage } & pT1a & $8(66.7)$ & $7(50)$ & \\
\hline & pT1b & $3(25)$ & $7(50)$ & \\
\hline & pТЗа & $1(8.3)$ & $(-)$ & \\
\hline \multicolumn{5}{|c|}{$\begin{array}{l}\text { LTPN/LRPN: Laparoscopic Transperitoneal/Retroperitoneal Partial Nephrectomy; SM: Surgical Margins, } \\
\text { RCC: Renal Cell Carcinoma; FG: Fuhrman Grade; Neg./Pos.: Negative/Positive; ccRCC: clear cell RCC; } \\
\text { pRCC: papillary RCC; chRCC: chromophobe RCC. } \\
{ }^{2} \text { Mann-Whitney test; 2-sided Fisher exact test; }{ }^{b} x^{2} \text { test, Fisher's test. }\end{array}$} \\
\hline
\end{tabular}

\section{Discussion}

In previous studies, the surgeon's decision to perform TP or RP approach was usually influenced by tumor's characteristics, patient's history of previous intraperitoneal operations and by his discretion and experience $(1,3,11$ $13,15,17,18)$. Previous studies demonstrated that both approaches have comparable outcomes or that LRPN outperforms LTPN in several parameters $(1,3,7,11-15,17$, 18). However, to the best of our knowledge, this is the first comparative study of TP and RP approach on pure LPN, exclusively for posterior, cTl RMs of similar complexity, in patients with similar demographic data. 
In our study, LRPN was associated with shorter OT. The difference in OT is probably due to the rapid access of LRPN to the tumor, avoiding bowel and extensive kidney mobilization, and due to the need for lysis of adhesions in the first cohort $(1,3,7,11-18)$. It is worth-mentioning that we noticed intrabdominal adhesions in more patients of the group A than anticipated by the history of previous intraperitoneal operation and this might prolong OT.

Commonly, WI is applied during PN in order to control bleeding, facilitating tumor excision and renorrhaphy and avoiding complications $(9,17,21-23)$. WIT below 20-25 minutes is generally considered safe (9). Efforts to minimize WIT resulted in the introduction of terms such as early unclamping technique, selective renal artery clamping technique, clampless PN and selective renal parenchymal ischemia $(9,16,22,23)$. We commonly occlude renal artery without occluding renal vein in both centers. Although the occlusion of both renal vessels reduces bleeding from the tumor bed and offers better visualization by preventing venous backflow, animal studies revealed that selective renal artery clamping is superior in preserving renal function postoperatively, as it allows the retrograde irrigation of the normal renal parenchyma by venous blood at lower oxygen tension (23). We used different techniques in order to achieve WI. As expected, group A (early unclamping technique) had shorter median WIT than group B. However, the difference was not statistically significant.

In cases where we met favorable conditions intraoperatively, we proceeded in clampless PN in order to complete resection and renal reconstruction. HD view and increased intrabdominal pressure due to pneumoperitoneum in laparoscopy facilitate clampless PN offering higher control of bleeding $(2,9)$. Solitary kidney model demonstrated lower incidence of Acute Kidney Injury and Chronic Kidney Disease when ischemia is not applied, although comparison of long-term results on renal function following on-clamp or off-clamp LPN did not demonstrate any benefit in favor of clampless technique in the case of normal contralateral renal function preoperatively $(21,23)$. In fact, all patients who underwent clampless LPN had no change in eGFR postoperatively. In any case, the possible benefit of clampless PN has to be balanced against the risk of intraoperative bleeding, which may affect the oncological outcome and the complication rate (9). Any other techniques except conventional WI are technically challenging and should not be popularized (5).

Transient vascular occlusion exposes the remnant kidney to WI-reperfusion injury, mitigating the renoprotective character of PN $(9,10,21-23)$. In addition, postoperative renal function is determined by the preoperative renal function, the volume of the resected RM and the suturing in order to perform renorrhaphy (22).

The variation of eGFR preoperatively and at discharge in each cohort results from the patients who received WI, as patients who underwent clampless LPN demonstrated no change in eGFR. However, we did not notice any benefit in favor of early unclamping technique. As tumor size and complexity are comparable between the two groups, this result may be due to the quality of renorrhaphy of each approach in posterior tumors or due to the quality of remaining parenchyma in each group $(24,25)$.

Usual intraoperative complications of LPN are bleeding and injuries of adjacent viscera (10). Bleeding (postoperative or delayed) and urine leak are the most common postoperative complications (10). Previous studies demonstrated that LRPN is related to lower intraoperative blood loss, as it provides excellent hilar control and demands lesser extent of dissection $(1,11,15)$.

The intraoperative transfusion rate of group $A$ and the fact that preoperative serum $\mathrm{Hb}$ value and variation of serum $\mathrm{Hb}$ value pre- and post-operatively were similar may confirm the result of previous studies. Nevertheless, this may be due to differences in clamping technique between cohorts (1). The majority of our complications were minor, while the only death was unrelated to the operation. An advantage of LRPN is the avoidance of peritoneal entry and bowel mobilization, resulting in earlier bowel recovery postoperatively $(1,7,15,19)$.

We did not observe any complications or readmissions related to bowel dysfunction in both cohorts, no patient needed antiemetic drugs postoperatively and we applied similar feeding protocols. In any case, intrabdominal adhesions following LTPN are usually of minor clinical importance $(1,14)$.

We did not notice difference in LOS, although previous studies favored LRPN $(1,3,7,12,14,15,17)$.

This result may be due to the application of similar perioperative care and discharge criteria, although different hospital settings and postoperative complications may affect outcome (11).

PSM rate was similar between approaches. PSM status was noticed only in patients who underwent clampless LPN and there may be a relationship between them. Our PSM rate is relatively high compared to previous studies, but this may be affected by the small sample size $(4,8,9$, $14,23)$. PSMs may increase the likelihood of recurrence, although their role in natural history of RCC is still under investigation and tumor's multifocality, grade and stage may be more important factors than PSM on the development of local recurrence (4). Nevertheless, microscopic PSMs do not seem to influence survival $(4,8)$.

Although our follow-up period is relatively short, none of the patients presented recurrence. In the cases of PSM, we schedule our patients in shorter follow-up time intervals, in order to manage a possible recurrence on time (8).

Our research has several limitations. The statistical power of the sample size is possibly capable to detect only the largest differences between cohorts. It was a retrospective study and patients were not randomly allocated to treatment groups. However, we believe that it is difficult to design randomized, prospective studies with larger sample to compare LTPN and LRPN exclusively for posterior RMs in order to draw definitive conclusions, as there are already a lot of approaches and there is a rapid progress in technology and surgical equipment.

Our results reflect the experience of high-volume laparoscopic surgeons and it may be difficult to replicate them in a different setting. Moreover, differences in hospital settings may affect outcomes. Finally, longer follow-up period is required for our functional and oncological results to mature. 


\section{Conclusions}

LRPN is associated with shorter OT compared to LTPN for patients with posterior cTl RMs. Ultimately, good results can be achieved with either approach in experienced hands and the choice should be based on surgeon's experience and judgment in order to achieve the optimum outcome for the patient.

\section{References}

1. Ren $T$, Liu $Y$, Zhao X, et al. Transperitoneal approach versus retroperitoneal approach: a meta-analysis of laparoscopic partial nephrectomy for renal cell carcinoma. PLoS One. 2014; 9:e91978.

2. Porpiglia F, Mari A, Bertolo R, et al. Partial nephrectomy in clinical T1b renal tumors: Multicenter Comparative Study of Open, Laparoscopic and Robot-assisted Approach (the RECORd Project). Urology. 2016; 89:45-51.

3. Ouzaid I, Xylinas E, Pignot G, et al. Laparoscopic partial nephrectomy: is it worth still performing the retroperitoneal route? Adv Urol. 2012; 2012:473457.

4. Minervini A, Campi R, Sessa F, et al. Positive surgical margins and local recurrence after simple enucleation and standard partial nephrectomy for malignant renal tumors: systematic review of the literature and meta-analysis of prevalence. Minerva Urol Nefrol. 2017; 69:523-38.

5. Marconi L, Challacombe B. Robotic partial nephrectomy for posterior renal tumours: retro or transperitoneal approach? Eur Urol Focus. 2018; 4:632-635.

6. Paulucci DJ, Beksac AT, Porter J, et al. A multi-institutional propensity score matched comparison of transperitoneal and retroperitoneal partial nephrectomy for cT1 posterior tumors. J Laparoendosc Adv Surg Tech A. 2019; 29:29-34.

7. Fu J, Ye S, Ye HJ. Retroperitoneal versus transperitoneal laparoscopic partial nephrectomy: a systematic review and meta-analysis. Chin Med Sci J. 2015; 30:239-44.

8. Vartolomei MD, Foerster B, Kimura S, et al. Oncologic outcomes after minimally invasive surgery for cT1 renal masses: a comprehensive review. Curr Opin Urol. 2018;28:132-138.

9. Mearini L, Nunzi E, Vianello A,et al. Margin and complication rates in clampless partial nephrectomy: a comparison of open, laparoscopic and robotic surgeries. J Robotic Surg. 2016; 10:135-44.

10. Zhao PT, Richstone L, Kavoussi LR. Laparoscopic partial nephrectomy. Int J Surg. 2016; 36:548-553.

11. Gin GE, Maschino AC, Spaliviero M, et al. Comparison of perioperative outcomes of retroperitoneal and transperitoneal minimally invasive partial nephrectomy after adjusting for tumor complexity. Urology. 2014; 84:1355-1360.

12. Fan $X, X u K$, Lin $T$, et al. Comparison of transperitoneal and retroperitoneal laparoscopic nephrectomy for renal cell carcinoma: a systematic review and meta-analysis. BJU Int. 2013; 111:611-621.

13. Muñoj-Rodriguez J, Prera A, Dominguez A, et al. Laparoscopic partial nephrectomy: comparative study of the transperitoneal pathway and the retroperitoneal pathway. Actas Urol Esp. 2018; 42:273-279.

14. Marszalek M, Chromecki T, Al-Ali BM, et al. Laparoscopic partial nephrectomy: a matched-pair comparison of the transperitoneal versus the retroperitoneal approach. Urology. 2011; 77:109-13.

15. Wright JL, Porter JR. Laparoscopic partial nephrectomy: comparison of transperitoneal and retroperitoneal approaches. J Urol. 2005; 174: 841-845.
16. Rassweiler JJ, Klein J, Tschada A, Gözen AS. Laparoscopic retroperitoneal partial nephrectomy using an ergonomic chair: demonstration of technique and matched-pair analysis. BJU Int. 2017; 119:349-357.

17. Ng CS, Gill IS, Ramani AP, et al. Transperitoneal versus retroperitoneal laparoscopic partial nephrectomy: patient selection and perioperative outcomes. J Urol. 2005; 174:846-849.

18. Kieran K, Montgomery JS, Daignault S, et al. Comparison of intraoperative parameters and perioperative complications of retroperitoneal and transperitoneal approaches to laparoscopic partial nephrectomy: Support for a retroperitoneal approach in selected patients. J Endourol. 2007; 21:754-9.

19. Klap J, Butow Z, Champy CM, et al. 1,000 retroperitoneoscopic procedures of the upper urinary tract: analysis of complications. Urol Int. 2019; 102:406-412.

20. Shefler A, Ghazi A, Zimmermann R, Janetschek G. Renal hilus clamping with tourniquet during laparoscopic partial nephrectomy. BJU Int. 2011; 107:1688-93.

21. Shah PH, George AK, Moreira DM, et al. To clamp or not to clamp? Long-term functional outcomes for elective off-clamp laparoscopic partial nephrectomy. BJU Int. 2016; 117:293-9.

22. Bagheri F, Puztai C, Farkas L, et al. Impact of parenchymal loss on renal function after laparoscopic partial nephrectomy under warm ischemia. World J Urol. 2016; 34:1629-1634.

23. Ng AM, Shah PH, Kavoussi LR. Laparoscopic partial nephrectomy: a narrative review and comparison with open and robotic partial nephrectomy. J Endourol. 2017; 31:976-984.

24. Corongiu E, Grande P, Di Santo A, et al. Safety and efficacy of retroperitoneal sutureless zero ischemia laparoscopic partial nephrectomy for low nephrometry score masses. Arch Ital Urol Androl. 2019;91:157-162.

25. Tiscione D, Cai T, Luciani LG, et al. Sutureless laparoscopic partial nephrectomy using fibrin gel reduces ischemia time while preserving renal function. Arch Ital Urol Androl. 2019; 91:30-34.

\author{
Correspondence \\ Nikolaos Ferakis, MD \\ ferakis@otenet.gr \\ Antonios Katsimantas, MD \\ antonioskatsimantas@gmail.com \\ Spyridon Paparidis, MD \\ sppap1986@gmail.com \\ Department of Urology, Korgialenio-Benakio Hellenic Red Cross Hospital, \\ Athens (Greece) \\ Nikolaos Charalampogiannis, MD \\ nickharas@yahoo.gr \\ Jens Rassweiler, $\mathrm{MD}$ \\ jens.rassweiler@slk-kliniken.de \\ Ali S Gozen, MD (Corresponding Author) \\ asgozen@yahoo.com \\ Department of Urology, SLK-Kliniken Heilbronn, University of \\ Heidelberg, Heilbronn, Germany
}

